\title{
Diagnostic des myopathies en Afrique de l'Ouest : expérience de quatre pays
}

\author{
Maroufou Jules Alao, Francis Lalya, Constant Adjien, Marius Adjagba, Simon Azombakin
}

Les maladies neuromusculaires sont des affections ubiquitaires et donc normalement présentes dans toutes les communautés ethniques et sous toutes les latitudes [1]. Elles sont toutefois très peu rapportées en Afrique, notamment dans sa partie sub-saharienne, hormis en Afrique du Sud où elles font l'objet de publications [2]. Cette situation est due aux difficultés de reconnaissance des cas, de confirmation diagnostique et surtout au poids encore très élevé des maladies infectieuses et des conséquences de la malnutrition dans beaucoup de ces pays [3]. Des professionnels de santé africains, avec l'aide des leurs homologues français tentent depuis 2009 d'apporter des aides au diagnostic et à la prise en charge de ces maladies. Le ROAMY (Réseau Ouest-Africain de Myologie) est destiné à encourager les initiatives dans ce domaine.

Les auteurs souhaitaient faire le point de cette expérience à travers les aspects épidémiologiques et diagnostiques d'une part, et d'autre part essayer de dégager des actions pour l'amélioration des interventions à visée thérapeutique.

Des patients suspects d'affections neuromusculaires ont été recrutés lors de consultations de myologie, sur la période allant de 2009 à 2015 . Les consultations étaient permanentes au Bénin dans le cadre d'une consultation de génétique médicale dans le Service de Pédiatrie et de Génétique Médicale du $\mathrm{CHU}$ de Cotonou [4]. Elles étaient foraines dans les autres pays que sont le Burkina Faso, la Guinée-Conakry et le Niger. Chaque patient adressé pour suspicion de myopathie bénéficiait d'un examen clinique complet. Les cas compatibles avec une myopathie (existence d'une histoire familiale, troubles ou perte de la marche, amyotrophie) étaient prélevés pour le dosage de la créatine phosphokinase (CPK) sérique. Lorsque c'était possible et nécessaire, un électroneuromyogramme était réalisé. Ce bilan était complété par des examens de génétique moléculaire avec extraction d'ADN dans le laboratoire de cytogénétique et de biologie moléculaire de la Faculté des Sciences de la Santé de Cotonou. S'ensuivait une recherche mutationnelle dans les gènes d'intérêt dans les laboratoires de génétique des Hôpitaux Cochin, Robert Debré et Salpêtrière de Paris en France [5, 6]. Au Bénin, au Burkina Faso, au Niger et en Guinée-Conakry, sur les 75, 20, 17 et 14 personnes adressées pour myopathie, respectivement 40, 15, 7 et 2 avaient un tableau clinique compatible avec une maladie neuromusculaire. Le taux de confirmation moléculaire a été respectivement de 22/40, $5 / 15,2 / 7$ et $0 / 2$ pour les malades vus au Bénin, au Burkina Faso, au Niger et en Guinée avec un taux de confirmation globale de 29/64 soit 45,31\%. Les pathologies retrouvées étaient la myopathie de Duchenne ( $n=20)$, la gamma sarcoglycanopathie $(\mathrm{n}=2)$, l'amyotrophie spinale infantile $(n=4)$, la dysferlinopathie $(n=2)$ et la myotonie de Becker $(n=1)$ comme présenté sur la figure 1 .

Les consultations étaient foraines dans trois pays par manque de ressources humaines formées spécifiquement en myologie. En effet, la myologie est très peu enseignée aussi bien dans les études médicales de base que dans les spécialités de pédiatrie et de neurologie notamment de l'adulte. La morbidité et la mortalité dans ces trois pays sont encore dominées par des maladies infectieuses et des affections non transmissibles avec une forte proportion des maladies cardiovasculaires [7]. Le nombre de neurologues ou neuropédiatres reste très faible eu égard aux standards mondiaux en la matière. La confirmation diagnostique reste faible à cause des difficultés de sélection des cas et surtout par manque d'explorations à visée étiologique comme la biopsie musculaire. Il aurait fallu biopsier tous les cas compatibles, réaliser la recherche des enzymes musculaires et ainsi mieux orienter la recherche moléculaire, qui seule permet d'avoir un diagnostic précis et d'envisager sereinement un conseil génétique [8].

Les maladies neuromusculaires en Afrique de l'Ouest sont dominées par la myopathie de Duchenne. Le rendement diagnostic reste faible surtout dans les pays sans consultation permanente. Ce rendement peut être amélioré par la mise en place d'infrastructures permettant la réalisation des biopsies musculaires et leur analyse conséquente. Ce qui orientera au mieux la recherche moléculaire encore tributaire de la coopération Nord-Sud exemplaire dans le domaine de la myologie.

Diagnosis of myopathies in West Africa: experience of four countries 
Diagnostic des myopathies en Afrique de P'Ouest : expérience de 4 pays

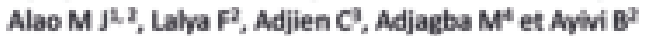

1 Senvice de Pidlatrie da OnU de la Mire et de Rintant-Lagane de Cotonou 2 Service de Ptdatrie et Gtnetique Midicale, CNHU de Cotonou 3 Service de Neurologie, CNivU de Cotonou 4 Laboratoire de gentipue humaine de la FSS, Cotonou, Benin

\section{Introduction}

Les myopathies sont très peu rapportées en Afrique en raison des difficultés de confirmation diagnostiques et rimportance encore élevées des maladies infectieuses. Des professionnels africains avec l'aide des praticiens français tentent depuis 2009 d'apporter des aides au diagnostic de ces maladies.

Objectif: Faire le point à travers les aspects épidémiologiques, diagnostiques et perspectives

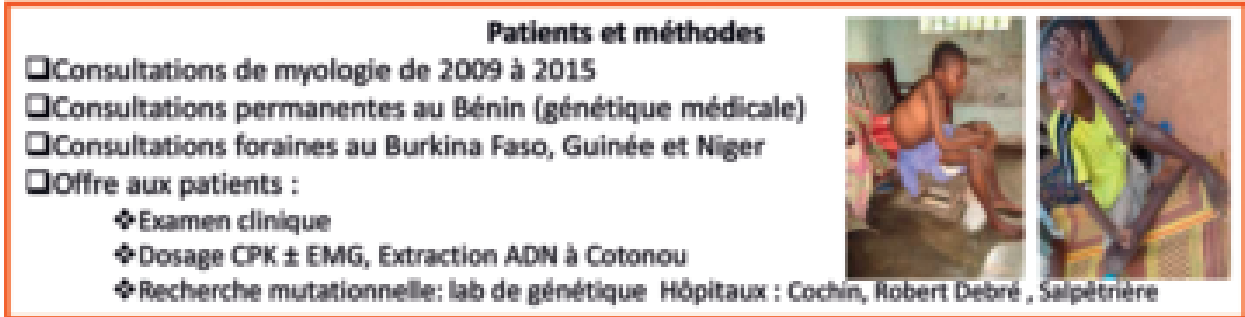

\section{Résultats}

\begin{tabular}{lccc}
\hline Pays & Vus & Suspectés & Confirmés \\
\hline Bénin & 75 & 40 & 22 \\
Burkina & 20 & 15 & 5 \\
Guinée & 14 & 2 & 0 \\
Niger & 17 & 7 & 2 \\
\hline
\end{tabular}

Pathologies retrouvées :

Dyopathie de Duchenne: 20

Gamma sarcoglycanopathie : 2

DAmyotrophie spinale infantile: 4

DDysferlinopathie: 2

aMyotonie de Becker : 1

Conclusion
Poursuite : diagnostic moléculaire
Perspectives : diagnostic biochimique par biopsie musculaires
et marquages immunohistochimiques voire western blot à Cotonou

Mot-cle : Mropathie, troubles de la marche, Duchenne, amyotrophie spinale infantile, dysferlincpathie

Remerclements a : TAFM, a FITIMA, au Dr Andoni Urtizberea et au Dr France Leturcq

LIENS D'INTÉRÊT

Les auteurs déclarent n'avoir aucun lien d'intérêt concernant les données publiées dans cet article.

\section{RÉFÉRENCES}

1. Mah JK, Korngut L, Dykeman J, et al. A systematic review and meta-analysis on the epidemiology of Duchene and Becker muscular dystrophy. Neuromuscul Disord 2014; 24 : 482-91.

2. Salih MA. Childhood muscular dystrophy: an African review. Ann Trop Paediatr 1985 ; 5 : 167-73.

3. Liu L, Oza S, Hogan D, et al. Global, regional, and national causes of child mortality in 2000-13, with projections to inform post-2015 priorities: an updated systematic analysis. Lancet $2015 ; 385: 430-40$
4. Alao MJ, Gangbo F, Lalèyè A, et al. Génétique clinique dans le Service de pédiatrie et de génétique médicale du Centre National Hospitalier et Universitaire de Cotonou : État des lieux et perspectives. Clin Mother Child Health 2011; 8 : 5-10.

5. Gangbo F, Lalèyè $\mathrm{A}$, Alao MJ, et al. Une décennie de pratique de caryotype au laboratoire de cytogénétique de la Faculté des Sciences de la Santé de Cotonou au Bénin : bilan et perspectives d'avenir. JAMO $2010 ; 4: 41-44$

6. Leturcq F, Tuffery-Giraud S. Aspects génétiques et moléculaires des dystrophinopathies. Arch Pediatr 2015; 22 : 12S3-11.

7. OMS. Statistiques sanitaires mondiales 2014. WHO/HIS/HSI/ 14.1.

8. Maulucci F, Tsouni P, Kuntzer T. Quand suspecter une myopathie? Forum Med Suisse 2015 ; 15 : 623-8. 